\section{Productivité de Plantations}

\section{d'épinette blanche}

\section{sur le sol sablonneux à Grand'Mère, P.Q.}

\author{
Par J. D. GAGNON \\ Le Service Canadien des Forêts \\ Sillery, P.Q.
}

"The Grand'Mere white spruce plantations, amongst the aldest and largest in Canada, are no longer stagnant."

\section{Résumé}

Une superficie d'environ neuf milles carrés, communément appelée "Proulx Block" dans les plantations d'épinette blanche à Grand-Mère, a été inventoriée en 1946, 1956, 1961 et 1966. Les résultats de ces inventaires démontrent que, sur environ $20 \%$ de la superficie caractérisée par le type forestier Calliergon, la croissance des arbres plantés en 1926 est des plus satisfaisantes; sur environ $45 \%$ de la superficie caractérisée par le type forestier Calliergon-P'olytrichum, la croissance est moyenne, mais environ $35 \%$ de la superficie caractérisée par le type forestier Cladonia-Polytrichum, la croissance progresse lentement.

L'auteur conclut que, en général, les plantations d'épinette blanche à Grand-Mère ne sont plus dans un état de dépérissement, et que la recrudescence de croissance, même dans le site le plus pauvre, est de bon augure.

The productivity of White Spruce Plantations on Sandy Soils at Grand'Mère, P.Q.
Abstract
About nine square miles of the Proulx Block white spruce plantations at Grand-Mère were in- ventoried in 1946,1956, 1961 and 1966. Results of these inventories show that on about $20 \%$ of the studied area characterized by the Calliergon forest type, growth of trees planted in 1926 is most satisfactory; on about $45 \%$ of the area char- acterized by the Calliergon-Polytrichum type, growth is fairly good; while on about $53 \%$ of the area characterized by the Cladonia-Polytrichum type, growth rate is slow.
It is concluded that, in general the growth of the white spruce plantations at Grand-Mère is no

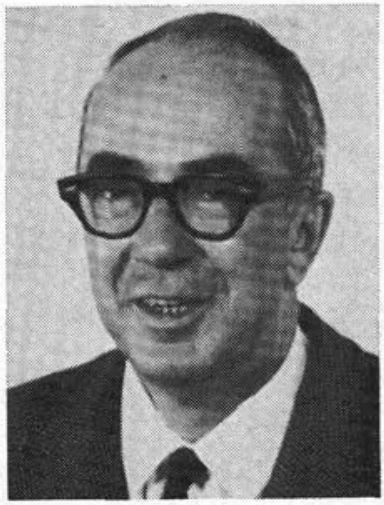

longer stagnant, and recovery, even on the poorest site, is encouraging.

\section{Introduction}

Le but de cette étude était de déterminer si les neuf milles carrés de plantations d'épinette blanche, établis à Grand'Mère entre les années 1925-1929 sur des dépôts sablonneux de la mer de Champlain, continuaient à dépérir.

Tous ceux qui ont eu à étudier ces plantations furent unanimes à reconnaître qu'elles étaient loin de fournir le rendement accru que l'on envisageait (Linteau, 1950; Cunningham, 1953; Goodacre et Coburn, 1957; Lafond, 1958; MacArthur, 1959; Paine, 1960; Swan, 1962). Pourtant, en dépit de l'état jugé déplorable de ces plantations, la Compagnie Consolidated Paper Corporation, aujourd'hui la Consolidated Bathurst Ltd., a tout a même pratiqué depuis 1953, en dehors des parcelles étudiées, des coupes d'éclaircies totalisant près de 45,000 cordes en 1968. Ces coupes se poursuivent sur une échelle importante sur tout le territoire de la plantation. De plus, Conway (1964) démontra la rentabilité des plantations de Grand'Mère en assurant à 50 ans un intérêt de $5 \%$ sur le coût de la mise de fonds.

En vue d'obtenir des informations précises sur le développement de ces plantations d'épinette blanche, les parcelles échantillonnées par Cunningham en 1946 ont été inventoriées en 1956 (Gagnon et MacArthur, 1959), et de nouveau en 1961 et 1966. Les résultats de ces quatre inventaires font l'objet de cette étude.

\section{Matériel et Méthode}

Nous avons suivi en 1956, 1961 et 1966, la procédure établie par Cunningham en 1946. Le dispositif d'inventaire se compose de 55 parcelles carrées d'un dixième d'acre chacune, systématiquement réparties à des intervalles de 10 chaînes de long de virées distantes de 20 chaînes. Ces parcelles, les 
mêmes que celles établies en 1946, avaient déjà été classifiées par types forestiers d'après les plantes indicatrices (Gagnon et MacArthur, 1959). Parmi les 55 parcelles étudiées, 4 ont été classées comme étant sur de bons sites Calliergon (figure 1), 25 sur des sites moyens du type Calliergon-Polytrichum (figure 2), et 26 sur des sites pauvres caractérisés par le type Cladonia-Polytrichum (figure 3). Egalement, sur une largeur d'environ trois chaînes tout le long de chacune des virées continues, nous avions procédé à une classification du milieu à l'aide de la végétation au sol, couvrant ainsi environ $5 \%$ de la superficie totale de la plantation. De cet inventaire, nour avions estimé qu'environ $20 \%$ de la superficie totale était caractérisée par le type forestier Calliergon, $45 \%$ par le type Calliergon-Polytrichum et $35 \%$ par le type Cladonia-Polytrichum.

Dans chaque parcelle, une courbe diamètre-hauteur a été tracée et le volume calculé. De plus, dans chaque parcelle, dix arbres dominants furent identifiés et leur hauteur et diamètre mesurés. Un trou dans le sol, d'un pied de profondeur, matérialise chaque coin de la parcelle -tudiée. Signalons incidemment que, dans les sols sablonneux, cette méthode est beaucoup plus rapide et plus durable que la méthode classique du piquet de coin.

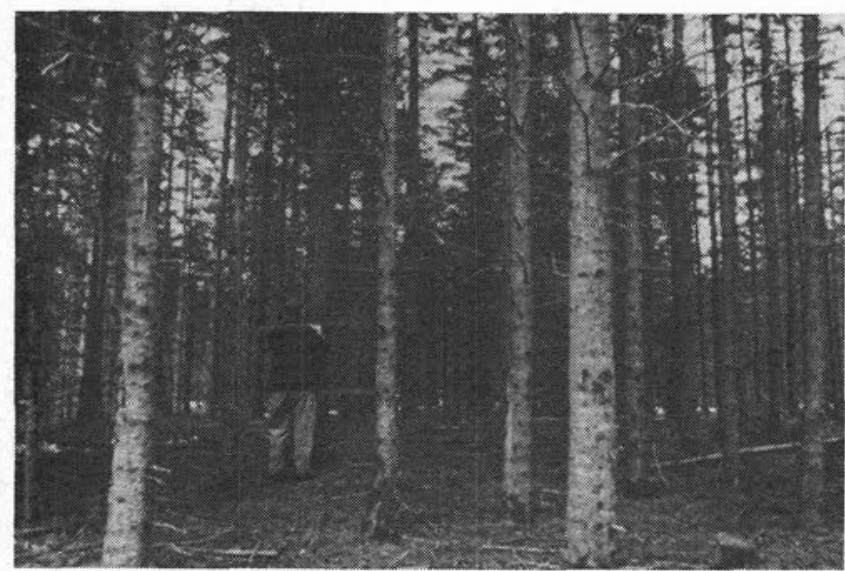

Fig. 1. Un bon site du type Calliergon. Les arbres sont à fût plein (non-tapering). La plaque blanche que l'observateur pose au d.h.p. a 6 pouces de largeur. Le diamètre moyen du peuplement est de 5.8 pouches.

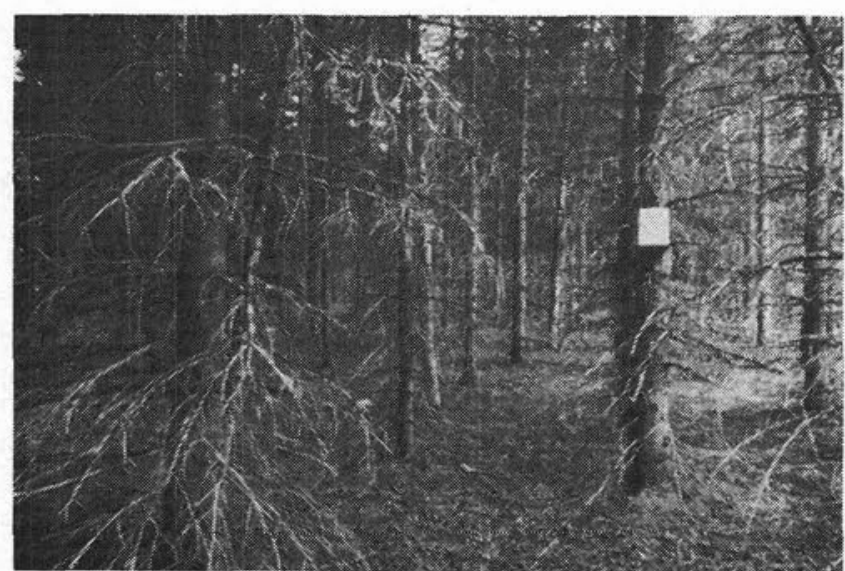

Fig. 2. Un site moyen du type Calliergon-Polytrichum. Les branches mortes sont abondantes et ramifiées tout le long du fût. Le diamètre moyen du peuplement est de 4.8 pouces.

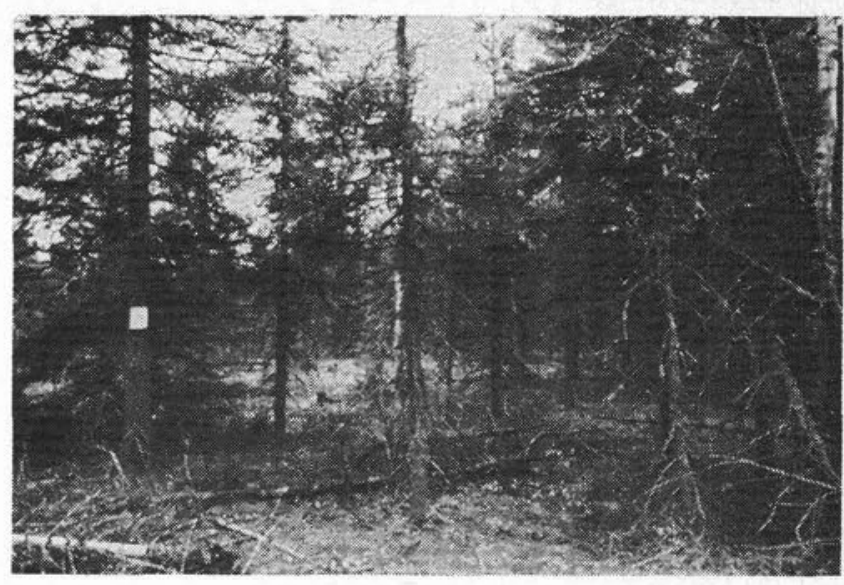

Fig. 3. Un site pauvre du type Cladonia-Polytrichum. Ces sites ne peuvent supporter qu'un nombre limité d'arbres et constituent la pierre d'achoppement des plantations de Grand'Mère. Le diamètre moyen du peuplement est de 3.9 pouces.

\section{Résultats}

Les résultats de ces inventaires successifs sont présentés en deux sections. La première traite du développement de la plantation (à différentes époques) classée par qualité de stations caractérisées par types forestiers; la seconde fait état de la croissance de dix arbres dominants de chaque parcelle étudiée dans chaque classe de productivité.

\section{Développement de la plantation}

Le tableau 1 montre, à différentes époques, le nombre d'arbres, la surface terrière, le diamètre moyen, le volume total à l'acre et l'accroissement annuel moyen de la plantation établie sur des sites

Tableau 1. Données sur la croissance en 1946, 1956, 1961 et 1966 des épinettes blanches plantées en 1926 et classées en 1956 dans trois types forestiers différents

\begin{tabular}{|c|c|c|c|c|}
\hline \multicolumn{2}{|l|}{ Types forestiers } & & & $\begin{array}{l}\text { Cla-Pol } \\
\text { (pauvre) }\end{array}$ \\
\hline \multirow{2}{*}{$\begin{array}{l}\text { Nombre de parcelles } \\
\text { Nombre d'arbres } \\
\text { plantés en } 1926 \\
\text { Survie en . . . . . . . . . }\end{array}$} & & 4 & 25 & 26 \\
\hline & $\begin{array}{l}1946^{*} \\
1956 \\
1961 \\
1966\end{array}$ & $\begin{array}{l}1,530 \\
1,350 \\
1,175 \\
1,080 \\
1,008\end{array}$ & $\begin{array}{r}1,530 \\
1,250 \\
874 \\
770 \\
720\end{array}$ & $\begin{array}{r}1,530 \\
1,130 \\
612 \\
565 \\
530\end{array}$ \\
\hline $\begin{array}{l}\text { Surface terrèire.... } \\
\text { (pieds carrés)....... }\end{array}$ & $\begin{array}{l}1946 \\
1956 \\
1961 \\
1966\end{array}$ & $\begin{array}{r}47.3 \\
127.0 \\
149.1 \\
183.0\end{array}$ & $\begin{array}{l}42.8 \\
62.5 \\
75.3 \\
91.7\end{array}$ & $\begin{array}{l}25.4 \\
28.0 \\
36.4 \\
44.8\end{array}$ \\
\hline $\begin{array}{l}\text { Diamètre moyen.... } \\
\text { (pouces) ........... }\end{array}$ & $\begin{array}{l}1946 \\
1956 \\
1961 \\
1966\end{array}$ & $\begin{array}{l}2.5 \\
4.4 \\
5.2 \\
5.8\end{array}$ & $\begin{array}{l}2.5 \\
3.6 \\
4.2 \\
4.8\end{array}$ & $\begin{array}{l}2.0 \\
2.9 \\
3.4 \\
3.9\end{array}$ \\
\hline $\begin{array}{l}\text { Volume total à l'acre. } \\
\text { (pieds cubes)........ }\end{array}$ & $\begin{array}{l}1946 \\
1956 \\
1961 \\
1966\end{array}$ & $\begin{array}{r}469 \\
1,940 \\
2,432 \\
3,044\end{array}$ & $\begin{array}{r}395 \\
732 \\
966 \\
1,226\end{array}$ & $\begin{array}{l}200 \\
268 \\
381 \\
517\end{array}$ \\
\hline $\begin{array}{l}\text { Volume marchand } \\
\text { à l'acre.......... }\end{array}$ & .1966 & 2,385 & 979 & 398 \\
\hline $\begin{array}{c}\text { Accroissement annuel } \\
\text { moyen (pieds cubes) }\end{array}$ & $\begin{array}{l}1946 \\
1956 \\
1961 \\
1966\end{array}$ & $\begin{array}{l}23 \\
65 \\
70 \\
76\end{array}$ & $\begin{array}{l}20 \\
24 \\
27 \\
31\end{array}$ & $\begin{array}{r}10 \\
9 \\
11 \\
13\end{array}$ \\
\hline Indice de site........ & .1966 & 50 & 40 & 30 \\
\hline
\end{tabular}

*Les valeurs pour l'année 1946 ont été extraites de l'inventaire de Cunningham 
bons, moyens et pauvres caractérisés respectivement par les types forestiers Calliergon, CalliergonPolytrichum et Cladenia-Polytrichum. Le volume marchand lors du dernier inventaire ainsi que l'indice de site à 50 ans apparaissent également au tableau 1. Le fait saillant qui se dégage de ce tableau est le regain d'accroissement qui se manifeste dans le site le plus pauvre.

\section{Croissance des arbres dominants}

Le tableau 2 montre la croissance moyenne de 10 arbres dominants ainsi que leur taux de mortalité dans chacune des trois qualité de stations étudiées. La quasi-uniformité du rythme d'accroissement en diamètre et en hauteur dans les trois sites est frappante. En effet, indépendamment des sites et des époques, il se fixe à environ 0.6 pouce pour le diamètre et à environ quatre pieds pour la hauteur. Le faible taux de mortalité est aussi digne de mention.

Tableau 2. Croissance moyenne de 10 arbres dominants de chaque parcelle étudiée dans différents types forectiers

\begin{tabular}{|c|c|c|c|}
\hline Types forestiers & Calliergon & Cal-Pol & Cla.Pol \\
\hline $\begin{array}{l}\text { Nombre de parcelles } \\
\text { Diamètre moyen ...... } 1956 \\
\quad 1961 \\
\text { (pouces) . . . . . . . } 1966\end{array}$ & $\begin{array}{l}4 \\
6.5 \\
7.1 \\
7.8\end{array}$ & $\begin{array}{l}25 \\
5.2 \\
5.9 \\
6.5\end{array}$ & $\begin{array}{l}26 \\
4.2 \\
5.1 \\
5.6\end{array}$ \\
\hline $\begin{array}{r}\text { Hauteur moyenne. . . } 1956 \\
1961 \\
\text { (pieds)............ } 1966\end{array}$ & $\begin{array}{l}35.5 \\
40.4 \\
44.2\end{array}$ & $\begin{array}{l}27.1 \\
31.2 \\
34.7\end{array}$ & $\begin{array}{l}20.4 \\
24.5 \\
28.0\end{array}$ \\
\hline $\begin{array}{cc}\text { Mortalité ....... } \\
(\%) & 1956-1961 \\
1961-1968\end{array}$ & $\begin{array}{l}0 \\
0\end{array}$ & $\begin{array}{r}1.2 \\
.8\end{array}$ & $\begin{array}{l}2.8 \\
1.6\end{array}$ \\
\hline
\end{tabular}

\section{Discussion}

A la lumière des travaux antérieurs et surtout de ceux de Linteau (1950), Lafond (1958), MacArthur (1959), Paine (1960) et Swan (1962), nous croyons que le taux élevé de mortalité depuis 1926 jusqu'à 1956, surtout dans le site le plus pauvre, est tout simplement dû à l'incapacité du site à satisfaire les exigences nutritives des arbres, lesquelles exigences s'accentuent avec la croissance. II a d'aillurs été démonté par Gagnon et MacArthur (1959) que, sur les sols sablonneux de Grand'Mère, de toutes les propriétés physiques des sols étudiés, seule l'épaisseur de l'humus de l'horizon Ap pouvait être reliée à la productivité des stations. En 1966 , l'épaisseur de cet humus était de $3.2,1.4$ et 0.9 centimètres sur les sites bons, moyens et pauvres. II importe de souligner que, en 1926, les plantations furent établies sur des sols déjà épuisés par l'agriculture et caractérisés en général par l'absence complète d'humus. La présence d'humus, après 40 ans, indique que la réversion de sols agricoles vers des sols forestiers s'est opérée graduellement et que les propriétés physico-chimiques des sols se sont sensiblement améliorées. La croissance satisfaisante de plantations effectuées depuis 1960 par nous-même sur des sites pauvres en témoignent.

Malgré une forte diminution dans le nombre d'arbres, on constate néanmoins une augmentation sensible de la surface terrière, du diamètre moyen et du volume total et marchand à l'acre qui, en s'ac- centuant avec le niveau de fertilité des stations étudiées et l'âge de la plantation, laisse suppose une certaine vigueur chez les arbres vivants. L'augmentation considérable entre 1961 et 1966 du volume total/acre, de l'ordre de $25 \%$ dans les bons sites, de $27 \%$ dans les sites moyens et de $36 \%$ dans les sites les plus pauvres est significative et laisse présager pour les années à venir, surtout dans le site le plus pauvre, une recrudescence de croissance des plus encourageantes.

Dans les bons sites du type Calliergon, l'accroissement moyen annuel de 76 pieds cubes à l'acre dépasse en 1966 le maximum indiqué par Vézina et Linteau (1968) pour les meilleurs peuplements naturels de sapin et d'épinette à 40 ans, et dépasse aussi celui obtenu à Petawawa par Stiell et Berry (1967) pour des plantations vigoureuses d'épinette blanche ayant, à 40 ans, un indice de site de 40 pour le même espacement, soit $5 \times 5$ pieds. Dans les sites moyens du type Calliergon-Polytrichum, un accroissement annuel moyen de 31 pieds cubes à l'acre avec 720 arbres peut se comparer assez bien avec les résultats de Stiell et Berry (1967) qui, pour un nombre d'arbres et volume/acre deux fois plus grand, montrent un accroissement annuel moyen également deux fois plus grand. Quant aux sites du type Cladonia-Polytrichum, les plus pauvres dans les plantations de Grand'-Mère, on remarque, après un ralentissement d'accroissement in 1956 un regain d'accroissement en 1961 qui se continue en 1966. L'indice de site de ces dernières stations se situe en dehors de la limite inférieure des classes de site trouvées par Linteau (1958), et par Stiell et Berry (1967). Ces cites pauvres constituent la pierre d'achoppement des plantations de Grand'Mère, et les efforts accomplis récemment par la Consolidated Bathurst Ltd. pour en tirer le meilleur profit possible sont louables. En effet, là où apparaît une régénération de sapins, on y pratique une coupe à blanc et au besoin on $y$ joint l'épinette noire. II y aurait peut-être avantage sur certains sites pauvres à garder les dominants comme semenciers afin de favoriser l'éclosion de la régénération abondante en épinettes blanches. Nous croyons que la texture sablonneuse des sols de Grand'Mère, plus riches en 1968 qu'en 1926, favoriserait mieux l'épinette blanche que le sapin.

\section{Croissance des arbres dominants}

Les données consignées au tableau 2 présentent une image plus précise du développement des plantations de Grand'Mère et mettent en évidence l'opinion déjà émise que, dans les stations les plus pauvres, le site ne pouvait supporter qu'un nombre limité d'arbres. Ces arbres accaparent le site au détriment des autres qui sont rapidement éliminés et qui en se décomposant satisfont aux exigences nutritives des arbres les plus vigoureux. Indépendamment de la qualité de la station, tous les arbres choisis parmi les dominants dans chaque parcelle se sont développés, de 1961 à 1966 au même rythme: en cinq ans, le diamètre s'est accru de 0.6 pouce environ et la hauteur d'environ quatre pieds. Entre 
1956 et 1961 , le pourcentage de mortalité chez ces arbres de choix s'accroît avec la pauvreté du site mais décroit depuis 1961. Cette diminution du taux de la mortalité et l'accroissement soutenu en diamètre et hauteur des arbres dominants indiquent que dans les sites les plus pauvres la période de stagnation est dépassée.

\section{Conclusions}

Dans ces études successives effectuées en 1946 , 1956, 1961 et 1966 dans les plantations de Grand'Mère, établies en 1926 sur des sols sablonneux, il ressort que:

1. En général, les plantations d'épinette blanche à Grand'Mère ne sont plus dans cet état de dépérissement que des travaux antérieurs rapportaient.

2. Sur environ $65 \%$ de la superficie, soit sur les types à Calliergon et à Calliergon-Polytrichum, le volume sur pied en 1966 surpasse ou égale celui que l'on retrouve dans les plantations vigoureuses d'épinette blanche de la région forestière des Grand-Lacs.

3. Sur environ $35 \%$ de la superficie, soit sur le type Cladonia-Polytrichum le volume sur pied en 1966

\section{References}

CONWAY, J. M. 1964. On the rentability of the Grand'Mère plantations Commun. C.P.P.A., Montreal, March 1964.

CUNNINGHAM, C. C. 1953. Growth and development of coniferous plantations at Grand'Mère, P.Q. Silv. Res. Note For. Br. Can. No. 103.

GAGNON, J. D., et MacARTHUR, J. D., 1959. La végétion forestière comme indice de la qualité des stations dans les plantations d'épinettes blanches. Div. Rech. Syl. Me. Rech. No. 70 Canada Min. Nord Canad. et Res. Nat. Dir. Forêts.

1959. Thirty years of growth in white spruce plantations at Grand'Mère, P.Q. Dept. North. Affairs and Nat. Res. For. Br. Q-11, May 1959.

GOODACRE, E. S., and COBURN, F. S., 1957. Report on the field planted areas of the Grand'Mère plantations (part 1). Unpublished report to the Consolidated Paper Corporation.

LAFOND A. 1958. Les déficiences en potassium et magnésium des plantations de Pinus strobus, Pinus resinosa, et Picea glauca dans la Province de Québec. Contr. Fonds de Rech. For. Univ. Laval No. 1.

LINTEAU, A. 1958. Ecological Status of white spruce in Quebec. Dept. North Affairs and Nat. Res. For. Br. Q-55, May 1958. n'est pas impressionnant. Cependant une reprise de l'accroissement en volume depuis 1961 est de bon augure.

A en juger part le nombre des études faites dans les plantations de Grand'Mère, où l'on retrouve les essences suivantes: épinette blanche, épinette de Norvège, pin gris, pin rouge et pin sylvestre, on peut facilement conclure que ces plantations constituent un laboratoire en plein air. En effet, les connaissances acquises sur le développement de ces plantations ont, en quelque sorte, orienté les travaux de reboisement accomplis sur une partie des 1,500,000 acres de terres abandonnées dans le Québec.

\section{Remerciements}

Nous désirons exprimer notre vie reconnaissance à M. J. D. MacArthur qui a collaboré étroitement à la localisation et au mesurage des parcelles inventoriées. Nous voulons aussi souligner l'aide de notre collègue $M$. René Doucet lors de l'inventaire de 1966. Nous sommes également reconnaissants aux officiers de la Consolidated Bathurst Ltd. tout spécialement à $\mathrm{M}$. John $\mathrm{M}$. Conway, pour leur collaboration dans la poursuite de cette étude.

1950. Report on the Proulx plantations. Canada, Dept. Res. and Dev. For. Br. (unpublished $\mathrm{ms}$ ).

MacARTHUR, J. D. 1959. Growth of Jack, Red and Scots Pine and White Spruce plantations, 1922 to 1956 at Grand'Mère, Que. Woodlands Review, Pulp and Paper Magazine of Canada, Vol. 60, Convention 1959.

PAINE, L. A. 1960. Nutrient deficiencies and climatic factors causing low volume production and active deterioration in white spruce. Studies in forest pathology XXII. Publication No. 1067. Forest Biol. Div. Canada, Dept. Agric., Ottawa, Ont.

STIELL, W. M., and BERRY, A. B., 1967. White spruce plantation growth and yield at the Petawawa forest experiment station. For. Br. Dept. Publ. No. 1200, Canada Dept. of For. and Rural Develop.

SWAN, H. S. D. 1962. The mineral nutrition of the Grand'Mère plantations C.P.P.A. Woodlands Section Index No. 2139 (F-1).

VEZINA, P. E., and LINTEAU, A. 1968. Growth and yield of balsam fir and black spruce in Quebec. Canada Dept. For. and Rural Develop. Information Report Q-X-2, Quebec Laboratory. 